\title{
Indoor massive multiple-input multiple-output channel characterization and performance evaluation*
}

\author{
Jian-zhi $\mathrm{LI}^{\dagger 1}$, Bo AI ${ }^{\dagger \ddagger 1,2}$, Rui-si HE${ }^{1}$, Qi WANG ${ }^{1}$, Mi YANG ${ }^{1}$, Bei ZHANG ${ }^{1}$, \\ Ke GUAN ${ }^{1}$, Dan-ping HE${ }^{1}$, Zhang-dui ZHONG ${ }^{1}$, Ting ZHOU ${ }^{3}$, Nan $\mathrm{LI}^{4}$ \\ $\left({ }^{1}\right.$ State Key Laboratory of Rail Traffic Control and Safety, Beijing Jiaotong University, Beijing 100044, China) \\ ( ${ }^{2}$ Engineering University of Armed Police Force, Telecommunication Engineering Department, Xi'an 710086, China) \\ $\left({ }^{3}\right.$ Key Laboratory of Wireless Sensor Network and Communication, Shanghai Institute of Microsystem and \\ Information Technology, Chinese Academy of Sciences, Shanghai 200050, China) \\ ( ${ }^{4}$ ZTE Corporation, Beijing 100191, China) \\ ${ }^{\dagger}$ E-mail: 15111063@bjtu.edu.cn; boai@bjtu.edu.cn
}

Received Jan. 8, 2017; Revision accepted Apr. 8, 2017; Crosschecked May 25, 2017

\begin{abstract}
We present a measurement campaign to characterize an indoor massive multiple-input multiple-output (MIMO) channel system, using a 64-element virtual linear array, a 64-element virtual planar array, and a 128-element virtual planar array. The array topologies are generated using a 3D mechanical turntable. The measurements are conducted at 2, 4, 6, 11, 15, and $22 \mathrm{GHz}$, with a large bandwidth of $200 \mathrm{MHz}$. Both line-of-sight (LOS) and non-LOS (NLOS) propagation scenarios are considered. The typical channel parameters are extracted, including path loss, shadow fading, power delay profile, and root mean square (RMS) delay spread. The frequency dependence of these channel parameters is analyzed. The correlation between shadow fading and RMS delay spread is discussed. In addition, the performance of the standard linear precoder - the matched filter, which can be used for intersymbol interference (ISI) mitigation by shortening the RMS delay spread, is investigated. Other performance measures, such as entropy capacity, Demmel condition number, and channel ellipticity, are analyzed. The measured channels, which are in a rich-scattering indoor environment, are found to achieve a performance close to that in independent and identically distributed Rayleigh channels even in an LOS scenario.
\end{abstract}

Key words: Massive MIMO; Channel modeling; 5G; Shadow fading; Delay spread; Matched filter; Entropy capacity; Condition number; Channel ellipticity

CLC number: TN92

\section{Introduction}

$\ddagger$ Corresponding author

* Project supported by the National Natural Science Foundation of China (Nos. 61501020 and U1334202), the State Key Laboratory of Rail Traffic Control and Safety (No. RCS2016ZJ005), the National Key Research and Development Program (No. 2016YFB1200102-04), the China Postdoctoral Science Foundation (No. 2016M591355), the Fundamental Research Funds for the Central Universities (No. 2016JBZ006), the National HighTech R\&D Program (863) of China (No. 2014AA01A706), the National S\&T Major Project (No. 2016ZX03001021-003), and the Key Laboratory of Wireless Sensor Network and Communication, Chinese Academy of Sciences (No. 2013005)

(C) ORCID: Jian-zhi LI, http://orcid.org/0000-0002-1978-1105

(C)Zhejiang University and Springer-Verlag Berlin Heidelberg 2017
While the global commercialization of the longterm evolution (LTE) standard is just beginning to flourish (Astely et al., 2009; He et al., 2016a), research on fifth generation $(5 \mathrm{G})$ mobile communication systems has been carried out extensively (Andrews et al., 2014). Currently, 5G has become the focus of global industry, and the International Telecommunications Union (ITU) has confirmed the vision and schedule for 5G called IMT-2020. Massive multiple-input multiple-output (MIMO) is 
considered to be one of the key technologies for $5 \mathrm{G}$, as it can improve spectrum efficiency and enhance network coverage and system capacity (Boccardi et al., 2014; Larsson et al., 2014; Wang et al., 2014; Cai et al., 2015). The number of antennas used in massive MIMO systems is generally from tens to hundreds, which is much larger than that in a traditional base station (BS). In a massive MIMO system, more independent data streams can be transmitted between BS and the terminals, improving the spectrum efficiency exponentially (Ngo et al., 2013). In addition, massive MIMO can realize accurate 3D beamforming and dynamic user tracking, enhance network coverage, and reduce the interference from neighboring BSs. It can be widely used in a variety of complex environments ( $\mathrm{Ng}$ et al., 2012; Liu et al., 2014).

Current research on channels in massive MIMO is based mostly on a theoretical independent and identically distributed (i.i.d.) complex Gaussian assumption. It is assumed that the channel vectors become pairwise orthogonal as the number of antennas at BS increases to infinity (Rusek et al., 2013). However, the actual radio channels of communication environments are complex, especially in the rich scattering (He et al., 2012a; Guan et al., 2016), or dynamic propagation scenarios (Wei et al., 2011; He et al., 2012b; Guan et al., 2013b). To achieve the best spectrum utilization, massive MIMO systems working at the frequency bands below $6 \mathrm{GHz}$ are expected to support large coverage and provide a moderate transmission rate for user experience, whereas the systems working at the frequency bands above $6 \mathrm{GHz}$ are expected to serve in short-range communications and carry out wide-bandwidth and high-speed transmissions. Since the system performance can be seriously affected by the radio channels, in-depth investigations into channel behaviors for typical scenarios (e.g., indoor halls, theaters, railway stations (Guan et al., 2014a), and railways (Gao et al., 2010; Guan et al., 2013a; 2014b; Ai et al., 2014; 2015)) at different frequency bands are needed.

Wu et al. (2015) proposed a non-stationary wideband channel model for massive MIMO, and investigated the statistical properties of the channel model by numerical analysis; however, no measurement-based verification was done. To better understand massive MIMO radio channels, channel measurement, which is considered the most direct and effective approach for investigating propagation characteristics, is an urgent and important work at present. However, there are only a few measurement reports on massive MIMO channels. Janssen et al. (1996) conducted a measurement campaign, and compared the channel characteristics for 2.40, 4.75 , and $11.50 \mathrm{GHz}$ by evaluating the path loss, the root mean square (RMS) delay spread, and the coherence bandwidth. However, this measurement campaign focused only on the single-input singleoutput (SISO) channel. Poon and Ho (2003) presented measurement results from 2 to $8 \mathrm{GHz}$ in both line-of-sight (LOS) and non-LOS (NLOS) scenarios for both office and residential environments. The clustering phenomenon over a $6 \mathrm{GHz}$ bandwidth was investigated, but only for the MIMO channel. In recent years, only a few initial channel measurements of massive MIMO channels have been conducted. Hoydis et al. (2012) performed an outdoor measurement campaign at $2.6 \mathrm{GHz}$ with a bandwidth of $20 \mathrm{MHz}$, by using a scalable virtual antenna array consisting of up to 112 elements. The results showed that most of the theoretical benefits of massive MIMO can be observed over the measured channels. The system performances of massive MIMO based on outdoor channel measurement campaigns were evaluated in Gao et al. (2012; 2015), Payami and Tufvesson (2012), and Flordelis et al. (2015). The investigated frequency is $2.6 \mathrm{GHz}$ and the bandwidth is $50 \mathrm{MHz}$. It is concluded that in realistic propagation environments the theoretical advantages of massive MIMO can be harvested. However, one should note that all these initial measurements focus only on a single carrier frequency (e.g., 2.6 GHz). There have been little research on channel behaviors of massive MIMO at different frequency bands. In this paper, we describe an indoor measurement campaign for six different carrier frequencies. Two groups with different carrier frequencies are chosen. One group includes carrier frequencies of 2,4 , and $6 \mathrm{GHz}$, which cover a large frequency band below 6 $\mathrm{GHz}$. The other includes carrier frequencies of 11 , 15 , and $22 \mathrm{GHz}$, which cover a frequency band above $6 \mathrm{GHz}$. The measurement bandwidth used in the campaign is $200 \mathrm{MHz}$, which is larger than those used in most of the existing measurements reported in Gao et al. (2012; 2015) and Hoydis et al. (2012). The large bandwidth can provide a high delay resolution. The types of arrays used at BS include a virtual 
linear array and two virtual planar arrays. Both LOS and NLOS propagation scenarios are considered in the measurements.

\section{Measurement campaign}

\subsection{Measurement system}

The measurements are performed in the frequency domain using a broadband vector signal generator (R\&S SMW200A) and a high-performance vector signal analyzer (R\&S FSW). The block diagram of the measurement system is illustrated in Fig. 1. The 3D turntable at the transmitter (Tx) can help to generate different array topologies. In most of previous channel measurements, the measured bandwidths are limited, whereas the bandwidth reaches $200 \mathrm{MHz}$ in our measurements, guaranteeing a high resolution for the multipath delay. Different array topologies are used, including a 64-element linear array, a 64-element planar array, and a 128-element planar array. For the 64-element planar array, there are eight elements in each row and each column. For the 128-element planar array, there are 16 elements in each row and eight elements in each column.

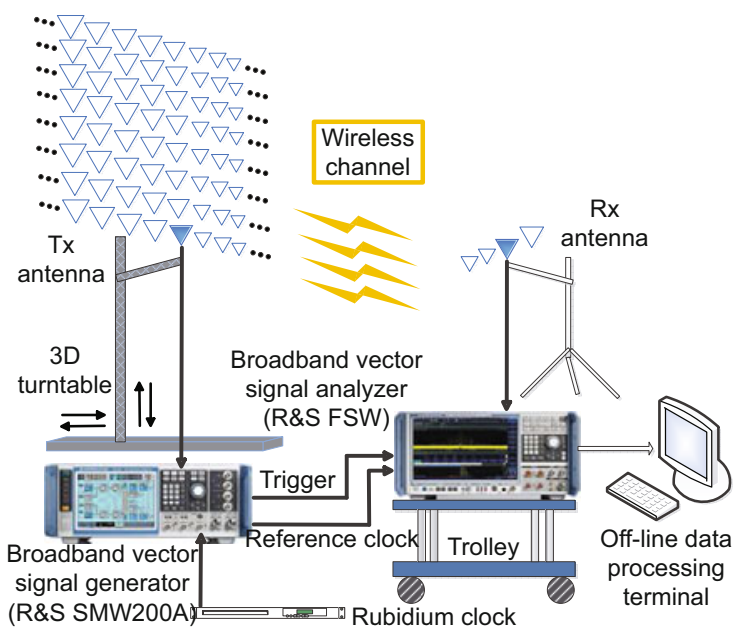

Fig. 1 Block diagram of the frequency domain measurement system (Tx: transmitter; $\mathbf{R x}$ : receiver)

The omnidirectional Tx antenna is moved along the guide rail track at BS to constitute the virtual antenna array during the measurement (Figs. 2a and $2 \mathrm{~b})$. The height of the Tx antenna from the guide rail is fully adjustable. By controlling a 3D turntable with a minimum step length of $0.1 \mathrm{~mm}$, a millimetergrade accuracy can be guaranteed. The receiver $(\mathrm{Rx})$
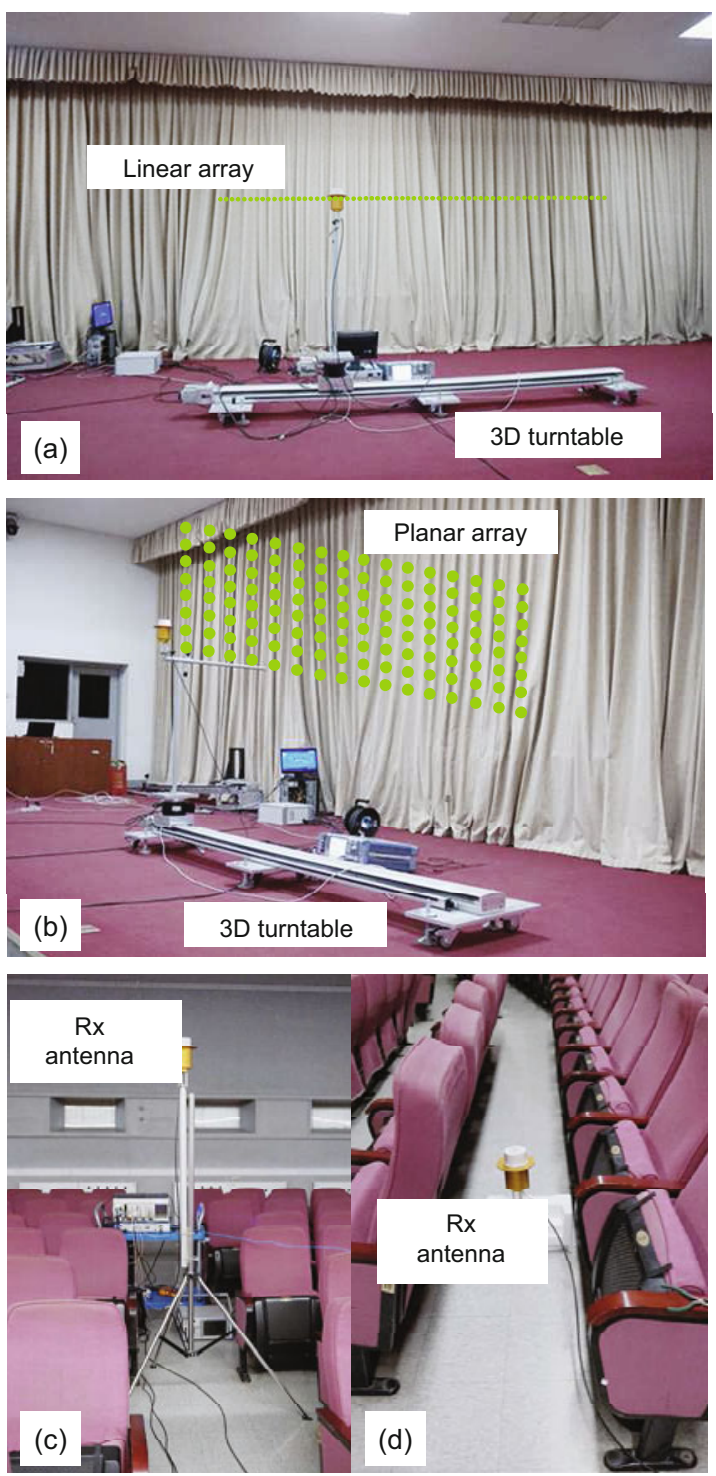

Fig. 2 The Tx antenna at the base station with a broadband vector signal generator and an $\mathrm{Rx}$ antenna with a broadband signal analyzer: (a) a virtual linear array with 64 elements (the corresponding $R x$ array is a 4-element virtual linear array); (b) a virtual planar array with $128(8 \times 16)$ elements; (c) an $R x$ antenna with a broadband signal analyzer in the LOS scenario; (d) an Rx antenna in the NLOS scenario (Tx: transmitter; Rx: receiver; LOS: line-of-sight; NLOS: none-LOS)

antenna is omnidirectional. It is carefully preset and located in the measurements. For measurements using a 64-element linear array and a 64-element planar array at $\mathrm{Tx}, \mathrm{Rx}$ is a 4 -element virtual array, whereas a single antenna is used on the Rx side when the 128-element planar array is used at Tx. For all array topologies, the distance between the adjacent antenna elements for both $\mathrm{Tx}$ and $\mathrm{Rx}$ is set to be at 
half a wavelength. The transmit power in the measurement is set to a constant value of $29 \mathrm{dBm}$ for all carrier frequencies. A total of 16 cycles of the transmitted signal are recorded for each subchannel.

\subsection{Measurement environment}

The measurements are conducted at the Center Hall in No. 9 Teaching Building, Beijing Jiaotong University, China. Part of our work was previously presented in Li et al. (2016) and Ai et al. (2017). The layout of the hall is illustrated in Fig. 3. The hall is $20.1 \mathrm{~m}$ long, $20.2 \mathrm{~m}$ wide, and $4.5 \mathrm{~m}$ high. To keep the channel as static as possible, special precautions are taken, such as measuring at midnight and avoiding any movement of people and other obstacles during the measurements. The $3 \mathrm{D}$ turntable is placed near the center of the platform, which is $0.8 \mathrm{~m}$ above the ground in the center hall. The height of the Tx antenna is $1.7 \mathrm{~m}$, and thus it is $2.5 \mathrm{~m}$ above the ground in the hall. Both the LOS and NLOS propagation scenarios are considered. Two placement strategies for the Rx antenna are designed for the LOS and NLOS scenarios, respectively. The Rx antenna is placed in the central corridor of the hall in the LOS scenarios, mounted at the top of a mast (Fig. 2c). The height of the Rx antenna from the ground is $2.0 \mathrm{~m}$ in this situation. In the NLOS scenarios, the Rx antenna is placed behind the seats (Fig. 2d), at a height of $0.5 \mathrm{~m}$ from the ground. The distance between the $\mathrm{Tx}$ and $\mathrm{Rx}$ is $15.0 \mathrm{~m}$ when the carrier frequency used in the measurements is 2,4 ,

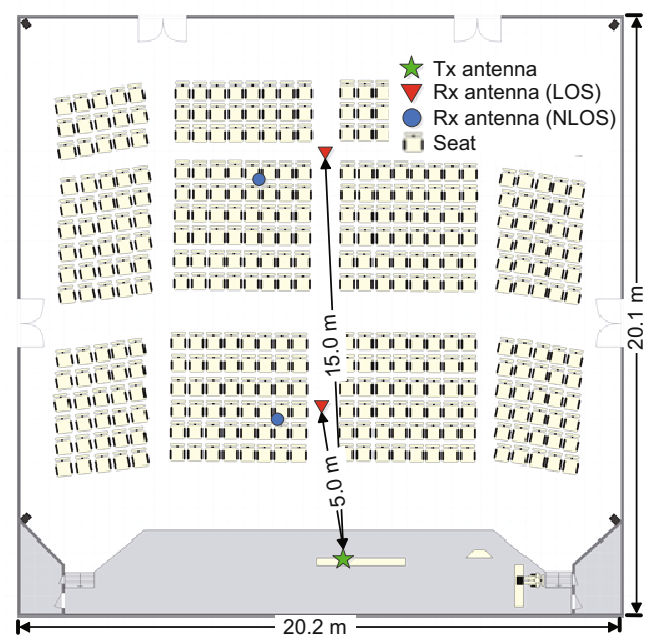

Fig. 3 Overview of the indoor measurement scenario (Tx: transmitter; $\mathrm{Rx}$ : receiver; LOS: line-of-sight; NLOS: none-LOS) or $6 \mathrm{GHz}$, while it is shortened to $5.0 \mathrm{~m}$ at 11,15 , or $22 \mathrm{GHz}$ due to higher path loss and the limited transmit power $(29 \mathrm{dBm})$. All clocks and sampling circuits in both the transmitter and receiver systems are slaved to the calibrated and coherent rubidium frequency standards.

\subsection{Calibration}

For all of the frequency bands investigated, the system has to be carefully calibrated before the measurements are conducted. To compensate for the influence of phase and amplitude variations caused by cables, amplifiers, and measurement equipment, the calibration is performed by using the received signal with the transmitter connected back-to-back with the receiver via a transmission cable as the reference. The received reference signal can be expressed as

$$
Y_{\mathrm{ref}}(f)=X(f) H_{\mathrm{Tx}}(f) H_{\mathrm{ref}}(f) H_{\mathrm{Rx}}(f),
$$

where $f$ is the frequency in the investigated bandwidth, $X(f)$ is the transmitted signal, $H_{\mathrm{Tx}}(f)$ and $H_{\mathrm{Rx}}(f)$ are the transfer functions of transmitter and receiver equipments, respectively, and $H_{\text {ref }}(f)$ can be regarded as a free-space transfer function. It is recognized that the back-to-back calibration omits the antenna characteristics from this adjustment. However, this back-to-back test is convenient and practical. Since both our Tx and Rx antennas have a $2 \mathrm{dBi}$ gain, this influence on the free-space transfer function $H_{\text {ref }}(f)$ can be compensated for conveniently in data pre-processing. The channel transfer functions $H(f)$ can be obtained from the measured in-phase and quadrature $(\mathrm{I} / \mathrm{Q})$ data. The data pre-processing method is the same as that in He et al. (2014), as follows:

$$
\begin{gathered}
Y(f)=X(f) H_{\mathrm{Tx}}(f) H(f) H_{\mathrm{Rx}}(f), \\
H(f)=\frac{Y(f)}{Y_{\mathrm{ref}}(f)} H_{\mathrm{ref}}(f) .
\end{gathered}
$$

Once $H(f)$ is obtained, we can obtain the channel impulse response (CIR), $h(\tau)$, by using the inverse discrete Fourier transform, as

$$
h(\tau)=\operatorname{IFFT}\left(H(f), N_{\mathrm{f}}\right)
$$

where $\tau$ is the delay of the received signal, and $N_{\mathrm{f}}=$ 513 is the number of measured frequency points. A Hann window is used to suppress the side lobes. 


\section{Channel characterization}

\subsection{Path loss and shadow fading}

Path loss is used to evaluate the propagation loss between the transmitter and the receiver $(\mathrm{He}$ et al., 2011; 2013). The local wideband path loss can be determined from the measured transfer function (He et al., 2014) as

$$
\mathrm{PL}=-10 \lg \left(\frac{1}{N_{\mathrm{f}}} \sum_{l=1}^{N_{\mathrm{f}}}\left|H\left(f_{l}\right)\right|^{2}\right),
$$

where $l$ is the index of the frequency point. To decrease the background noise, the arithmetic average is employed over 10 cycles of the received signal. Since in our measurements the distance between Tx and Rx is fixed, more attention should be paid to shadow fading, which is irrespective of distance. Shadow fluctuation on a decibel scale is modeled conventionally as a real zero-mean Gaussian process (Molisch, 2011):

$$
X_{\mathrm{S}} \sim N\left(0, \sigma_{\mathrm{S}}^{2}\right)
$$

where $N\left(0, \sigma_{\mathrm{S}}{ }^{2}\right)$ represents a Gaussian distribution with zero mean and standard deviation (STD) $\sigma_{\mathrm{S}}$. Shadow fading can be obtained by removing the distance dependence from the measured path loss. Table 1 summarizes the estimated $\sigma_{\mathrm{S}}$ for the three types of array topologies. In our measurements, the physical size in horizontal dimension of the antenna array at BS is usually large, especially at lower carrier frequencies (e.g., 2, 4, and $6 \mathrm{GHz}$ ). For example, a 128 -element planar array $(8 \times 16)$ is $1.12 \mathrm{~m}$ wide and $0.52 \mathrm{~m}$ high when the carrier frequency is $2 \mathrm{GHz}$, and a 64-element linear array takes up about $4.72 \mathrm{~m}$ in horizontal dimensions at the same frequency. The span of the array in the horizontal dimension is generally several times larger than half a wavelength. It is considered to be out of the stationary region $(\mathrm{He}$ et al., 2015a). For this reason, the data corresponding to the left and the right regions of the arrays is treated individually for comparison. Table 1 summarizes the estimated $\sigma_{\mathrm{S}}$ at six frequency bands in both the LOS and NLOS scenarios for three types of array topologies. The results are also shown in Fig. 4 for better visualization. In LOS scenarios, as the frequency increases, the estimated $\sigma_{\mathrm{S}}$ decreases in the overall trend. Fig. 4 a reflects this phenomenon more intuitively. We notice that the estimated $\sigma_{\mathrm{S}}$ 's
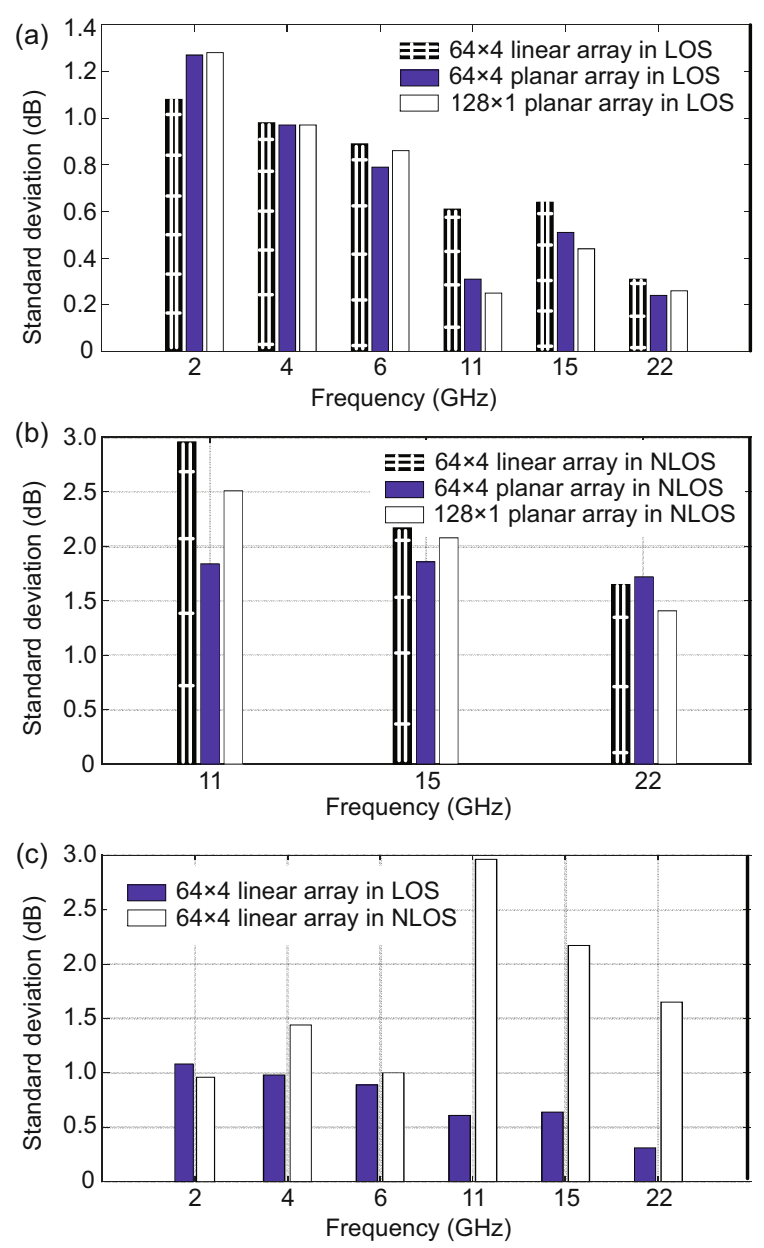

Fig. 4 Measured standard deviations for the three types of array topologies: (a) at 2, 4, 6, 11, 15, and $22 \mathrm{GHz}$ in the LOS scenario; (b) at 11, 15, and 22 GHz in the NLOS scenario; (c) at 2, 4, 6, 11, 15, and $22 \mathrm{GHz}$ for the 64-element linear array in both the LOS and NLOS scenarios (LOS: line-of-sight; NLOS: none-LOS)

at $2 \mathrm{GHz}$ in the NLOS scenarios for all the arrays are slightly smaller than those in the LOS scenarios, while the situation is just the opposite for other investigated frequencies. This phenomenon is probably due to the higher capacity of diffraction for lower frequencies. Moreover, difference of $\sigma_{\mathrm{S}}$ between the left and the right regions of the array can be observed, implying that the shadow fluctuation of the massive MIMO channel is non-stationary in the spatial domain. In addition, a clear dependency between $\sigma_{\mathrm{S}}$ and the investigated frequency is observed at frequencies above $6 \mathrm{GHz}$ (i.e., 11, 15, and $22 \mathrm{GHz}$ ) in the NLOS scenario (Fig. 4b). The overall estimated $\sigma_{\mathrm{S}}$ decreases with the increasing frequency in the NLOS scenarios. It is noted that the $\sigma_{\mathrm{S}}$ 's at these 
frequencies in the NLOS scenarios are much larger than those in the LOS scenarios. As an example, Fig. $4 \mathrm{c}$ shows a comparison of $\sigma_{\mathrm{S}}$ 's between LOS and NLOS scenarios for the 64 -element linear array. It is reasonable to see a large power variation in the NLOS scenarios, since most of the energy is from the LOS component at high frequencies.

\subsection{Power delay profile and RMS delay spread}

Power delay profile (PDP) is a basic channel parameter, which demonstrates the power distribution of the received signal under different time delays (He et al., 2016b). The average PDP (APDP) is generally used to calculate the delay spread and the coherence bandwidth. PDP can be obtained from the measured CIR, $h(\tau)$, as

$$
\operatorname{PDP}(\tau)=|h(\tau)|^{2} .
$$

To obtain the APDP which decreases the noise impact and avoids mistaking any receiver noise for actual multipath components, an arithmetic average is employed over 10 cycles of the measured PDP. Such a processing also ensures that the received multipath components have a sufficient SNR. To perform a quantitative analysis, a noise threshold, which is $6 \mathrm{~dB}$ above the noise floor (Molisch and Steinbauer, 1999), is set to eliminate the noise. The RMS delay spread, which is a characterization of channel delay dispersion, is estimated. It can be calculated as follows (Molisch, 2011):

$$
\tau_{\mathrm{rms}}=\sqrt{\frac{\sum_{p} \operatorname{APDP}\left(\tau_{p}\right) \tau_{p}^{2}}{\sum_{p} \operatorname{APDP}\left(\tau_{p}\right)}-\left(\frac{\sum_{p} \operatorname{APDP}\left(\tau_{p}\right) \tau_{p}}{\sum_{p} \operatorname{APDP}\left(\tau_{p}\right)}\right)^{2}},
$$

where $\tau_{p}$ and $\operatorname{APDP}\left(\tau_{p}\right)$ represent the delay and corresponding power of the $p$ th path, respectively.

The RMS delay spread corresponding to each position of the Tx array is estimated. Table 2 summarizes the mean STDs of the RMS delay spread for three types of array topologies in the measurements. The mean values of the RMS delay spread

Table 1 Standard deviations of the shadowing fluctuation at different frequencies in the LOS and NLOS

\begin{tabular}{|c|c|c|c|c|c|c|c|c|c|c|c|c|c|}
\hline \multirow{3}{*}{$\begin{array}{c}\text { Array } \\
\text { topology } \\
\text { at Tx }\end{array}$} & \multirow{3}{*}{$\begin{array}{l}\text { Region } \\
\text { of the } \\
\text { array }\end{array}$} & \multicolumn{12}{|c|}{ Standard deviation $(\mathrm{dB})$} \\
\hline & & \multicolumn{6}{|c|}{ Frequency in the LOS scenario $(\mathrm{GHz})$} & \multicolumn{6}{|c|}{ Frequency in the NLOS scenario $(\mathrm{GHz})$} \\
\hline & & 2 & 4 & 6 & 11 & 15 & 22 & 2 & 4 & 6 & 11 & 15 & 22 \\
\hline $64 \times 4$ & Left & 1.24 & 1.01 & 0.75 & 0.30 & 0.32 & 0.29 & 0.85 & 1.69 & 0.91 & 2.58 & 2.31 & 1.89 \\
\hline \multirow[t]{2}{*}{ linear } & Right & 0.78 & 0.94 & 0.80 & 0.36 & 0.45 & 0.16 & 0.97 & 1.13 & 1.04 & 1.92 & 1.91 & 1.36 \\
\hline & Overall & 1.08 & 0.98 & 0.89 & 0.61 & 0.64 & 0.31 & 0.96 & 1.44 & 1.00 & 2.96 & 2.17 & 1.65 \\
\hline \multirow{3}{*}{$\begin{array}{l}64 \times 4 \\
\text { planar }\end{array}$} & Left & 1.15 & 1.00 & 0.79 & 0.32 & 0.52 & 0.24 & 1.09 & 1.38 & 1.10 & 1.88 & 1.81 & 1.75 \\
\hline & Right & 1.35 & 0.86 & 0.83 & 0.30 & 0.49 & 0.23 & 1.07 & 1.30 & 1.14 & 1.70 & 1.79 & 1.68 \\
\hline & Overall & 1.27 & 0.97 & 0.79 & 0.31 & 0.51 & 0.24 & 1.09 & 1.31 & 1.10 & 1.84 & 1.86 & 1.72 \\
\hline \multirow{3}{*}{$\begin{array}{l}128 \times 1 \\
\text { planar }\end{array}$} & Left & 1.28 & 1.08 & 0.80 & 0.19 & 0.41 & 0.23 & 1.00 & 1.57 & 0.59 & 1.09 & 2.22 & 1.12 \\
\hline & Right & 1.29 & 0.83 & 0.91 & 0.30 & 0.38 & 0.28 & 0.89 & 0.94 & 0.68 & 1.27 & 0.99 & 1.50 \\
\hline & Overall & 1.28 & 0.97 & 0.86 & 0.25 & 0.44 & 0.26 & 0.95 & 1.57 & 0.64 & 2.51 & 2.08 & 1.41 \\
\hline
\end{tabular}
scenarios for the three types of array topologies

Tx: transmitter; LOS: line-of-sight; NLOS: none-LOS

Table 2 Means and standard deviations for the RMS delay spread at different frequencies in the LOS and

\begin{tabular}{|c|c|c|c|c|c|c|c|c|c|c|c|c|c|}
\hline \multirow{3}{*}{$\begin{array}{c}\text { Array } \\
\text { topology } \\
\text { at } \mathrm{Tx}\end{array}$} & \multirow{3}{*}{$\begin{array}{l}\text { Statistical } \\
\text { parameter }\end{array}$} & \multicolumn{12}{|c|}{ RMS delay spread (ns) } \\
\hline & & \multicolumn{6}{|c|}{ Frequency in the LOS scenario $(\mathrm{GHz})$} & \multicolumn{6}{|c|}{ Frequency in the NLOS scenario $(\mathrm{GHz})$} \\
\hline & & 2 & 4 & 6 & 11 & 15 & 22 & 2 & 4 & 6 & 11 & 15 & 22 \\
\hline $64 \times 4$ & Mean & 29.36 & 26.09 & 29.19 & 19.79 & 18.55 & 19.05 & 27.84 & 25.82 & 28.93 & 13.23 & 18.95 & 16.93 \\
\hline linear & STD & 4.09 & 3.20 & 4.06 & 3.15 & 2.86 & 2.16 & 3.85 & 4.75 & 3.40 & 4.36 & 5.29 & 4.51 \\
\hline $64 \times 4$ & Mean & 29.17 & 27.60 & 29.61 & 21.78 & 19.56 & 17.12 & 28.96 & 26.32 & 28.13 & 14.38 & 19.45 & 18.38 \\
\hline planar & STD & 4.30 & 3.90 & 3.30 & 2.72 & 3.51 & 1.93 & 3.78 & 3.82 & 4.08 & 4.39 & 4.94 & 4.92 \\
\hline $128 \times 1$ & Mean & 28.35 & 27.12 & 29.28 & 21.80 & 20.08 & 17.02 & 29.50 & 25.80 & 28.51 & 11.38 & 22.20 & 18.16 \\
\hline planar & STD & 4.21 & 3.59 & 3.59 & 2.33 & 2.92 & 2.33 & 3.97 & 4.57 & 3.67 & 3.43 & 4.87 & 4.18 \\
\hline
\end{tabular}
NLOS scenarios for the three types of array topologies 
are generally less than $30 \mathrm{~ns}$ (Table 2), which is in accordance with the classical measurement results for indoor environments (Molisch, 2011). The STDs of the RMS delay spread are larger than 2 ns in general. This reveals that the delay dispersion over the massive MIMO array is non-stationary in both horizontal and vertical dimensions. It can be further observed from Table 2 that the overall mean value of RMS delay spread at frequencies beyond $6 \mathrm{GHz}$ is smaller than that at frequencies below $6 \mathrm{GHz}$. The weak multipath components cannot be detected, resulting in a lower RMS delay spread. Moreover, a clear dependency between the mean value of the RMS delay spread and the carrier frequency is observed at all frequencies in the LOS scenarios (Fig. 5a). The means of the RMS delay spread decrease with the increase in frequency in the LOS scenarios for all arrays. However, in the NLOS scenarios, the means of the RMS delay spreads at these frequencies increase as the frequency increases
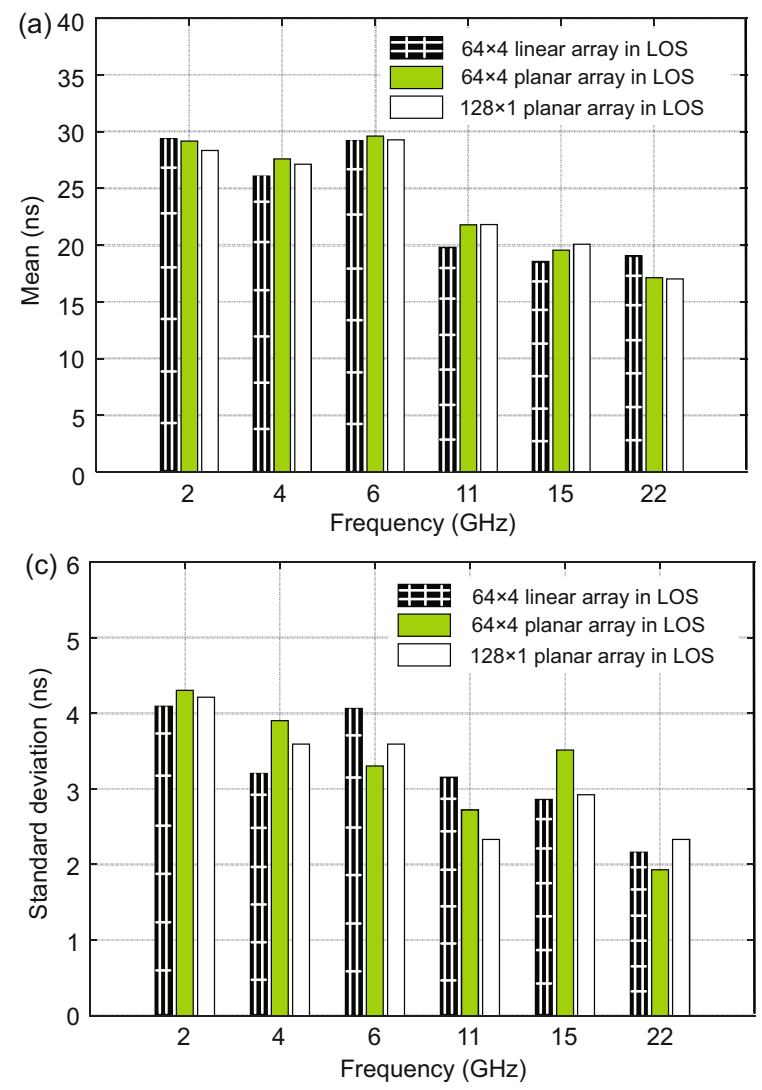

(Fig. 5b). In addition, a clear dependency between the STD of the RMS delay spread and the investigated frequency is found in the LOS scenarios for all arrays. The STD of the RMS delay spread decreases with the increase in frequency, as shown by the bar graphs in Fig. 5c. Nevertheless, the overall STD of the RMS delay spread at frequencies above $6 \mathrm{GHz}$ is much larger than that at frequencies below $6 \mathrm{GHz}$. As an example, Fig. 5d shows a comparison of the STDs of RMS delay spread between the LOS and NLOS scenarios at all frequencies for the 64-element planar array.

\subsection{Cross-correlation property}

The fluctuation rule of RMS delay spread is found to be similar to that of the estimated path loss. Feuerstein et al. (1994), Greenstein et al. (1997), and Salous and Gokalp (2007) conducted relevant research that relates RMS delay spread to path loss. The authors pointed out that RMS delay spread
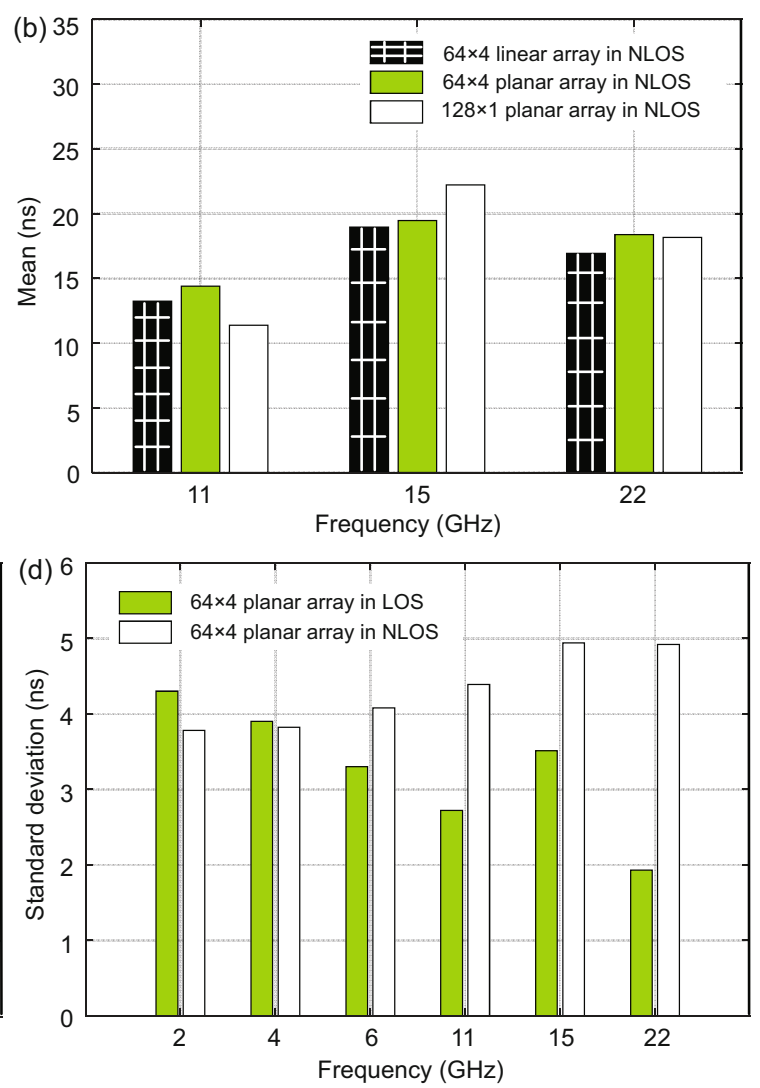

Fig. 5 Means and STDs of the RMS delay spread for three types of array topologies: (a) at 2, 4, 6, 11, 15, and $22 \mathrm{GHz}$ in the LOS scenario; (b) at 11, 15, and $22 \mathrm{GHz}$ in the NLOS scenario; (c) at 2, 4, 6, 11, 15, and $22 \mathrm{GHz}$ in the LOS scenario; (d) for a 64-element planar array in both the LOS and NLOS scenarios (STD: standard deviation; RMS: root mean square; LOS: line-of-sight; NLOS: none-LOS) 
increases exponentially with the path loss. In this study, we investigate the correlation between the fluctuation in RMS delay spread and shadow fading. To offset any potential effect of the distance between Tx and $\mathrm{Rx}$, the mean value of the total RMS delay spread is subtracted. Thus, the impact of the distance is removed. The correlation at $15 \mathrm{GHz}$ in the NLOS scenario for a 64-element planar array is illustrated in Fig. 6. The shadow fluctuations are plotted on the $X$ axis with the corresponding fluctuations in RMS delay spread on the $Y$ axis. Since the $\mathrm{Rx}$ antenna is a 4-element linear array, the data set for each antenna element is represented by one type of specific mark in Fig. 6.

We can clearly observe that the fluctuation in the RMS delay spread is positively correlated with shadow fading. It can be further observed from Fig. 6 that the correlation exists for all Rx antennas, since the distribution of each data set shows a good consistency. To give a quantitative analysis, the Pearson product-moment correlation coefficient, which is a

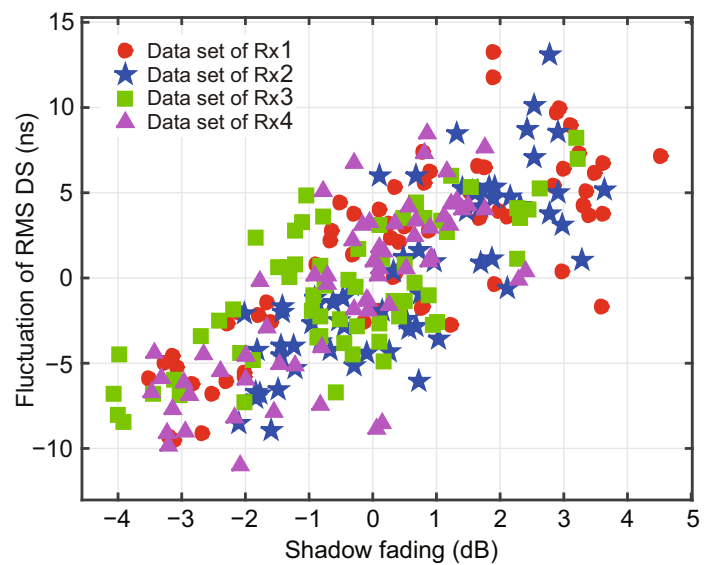

Fig. 6 Fluctuations in the RMS delay spread (DS) against shadow fading at $15 \mathrm{GHz}$ in the NLOS scenario for the 64-element planar array (RMS: root mean square; NLOS: none-line-of-sight; $\mathrm{Rx}$ : receiver) measure of the strength and direction of the linear relationship between two variables $X$ and $Y$, is calculated as (He et al., 2015b)

$$
\rho_{X, Y}=\frac{E[X Y]-E[X] E[Y]}{\sqrt{E\left[X^{2}\right]-E[X]^{2}} \sqrt{E\left[Y^{2}\right]-E[Y]^{2}}},
$$

where $E[\cdot]$ denotes the expected value, and $\rho_{X, Y}$ ranges from -1 to 1 . It is commonly assumed that $\left|\rho_{X, Y}\right|>0.50$ indicates a strong correlation. A linear correlation is validated when $\left|\rho_{X, Y}\right|=1.00$. Table 3 summarizes the calculated correlation coefficients between the fluctuation in the RMS delay spread and the shadow fading at different frequencies in the LOS and NLOS scenarios for three types of array topologies. In most cases, the calculated correlation coefficient is positive, indicating a positive correlation. A calculated negative correlation coefficient occurs only at a small number close to zero, which can be generally regarded as uncorrelated. As shown in Table 3, the overall value of the correlation coefficient is about 0.60 at frequencies below $6 \mathrm{GHz}$ in both the LOS and NLOS scenarios, which indicates a strong correlation. From a physics mechanism standpoint, an explanation of this phenomenon is that the detected multipath components with larger delays usually come from different propagation paths from the transmitter to the receiver, and experience more shadow effect and result in a larger fluctuation of received power. Since the multipath components would cause a delay spread of the transmitted signal, it is not surprising to observe a correlation between shadow fading and RMS delay spread. However, a different phenomenon can be observed at frequencies beyond $6 \mathrm{GHz}$ in an LOS scenario. The overall correlation coefficient becomes much weaker and even approaches zero (e.g., at $22 \mathrm{GHz}$ ), indicating no correlation. In contrast, an overall value of the correlation coefficient of 0.70 is found in the NLOS scenario,

Table 3 Correlation coefficients of shadow fading and fluctuations of RMS delay spread at different frequencies in the LOS and NLOS scenarios for three types of array topologies

\begin{tabular}{|c|c|c|c|c|c|c|c|c|c|c|c|c|}
\hline \multirow{3}{*}{$\begin{array}{c}\text { Array } \\
\text { topology } \\
\text { at Tx }\end{array}$} & \multicolumn{12}{|c|}{ Correlation coefficient } \\
\hline & \multicolumn{6}{|c|}{ Frequency in the LOS scenario $(\mathrm{GHz})$} & \multicolumn{6}{|c|}{ Frequency in the NLOS scenario $(\mathrm{GHz})$} \\
\hline & 2 & 4 & 6 & 11 & 15 & 22 & 2 & 4 & 6 & 11 & 15 & 22 \\
\hline $64 \times 4$ linear & 0.55 & 0.61 & 0.63 & 0.50 & 0 & 0 & 0.61 & 0.70 & 0.40 & 0.80 & 0.79 & 0.65 \\
\hline $64 \times 4$ planar & 0.68 & 0.70 & 0.58 & 0.19 & 0.50 & 0 & 0.63 & 0.52 & 0.69 & 0.79 & 0.77 & 0.54 \\
\hline $128 \times 1$ planar & 0.68 & 0.60 & 0.50 & 0 & 0.09 & 0 & 0.45 & 0.66 & 0.45 & 0.80 & 0.66 & 0.47 \\
\hline
\end{tabular}

Tx: transmitter; RMS: root mean square; LOS: line-of-sight; NLOS: none-LOS 
indicating a significant correlation. An explanation of this phenomenon is that the multipath components with larger delays become much weaker because of a higher path loss, and these weak multipath components cannot be detected when a strong and stable LOS component is present due to the limited dynamic range of the measurement system. In an NLOS scenario, the LOS component is much weaker and the multipath components with large delays are detectable, resulting in a strong correlation. The correlation coefficients obtained above are expected to be useful for the development of a massive MIMO channel simulator.

\section{Performance evaluation}

In this section we focus on the performance evaluation of the massive MIMO channel, and investigate that to which extent a theoretical performance can be achieved in realistic channels. The performance of the matched filter is investigated. Entropy capacity, Demmel condition number, and channel ellipticity based on measurement data are discussed.

\subsection{Matched filter precoding}

The multipath effect of the radio channel can cause delay dispersion, which further leads to intersymbol interference (ISI) in wireless communication systems (Molisch, 2011). Payami and Tufvesson (2013) investigated the delay spread reduction using standard massive MIMO precoders, including matched filter $(\mathrm{MF})$, zero forcing $(\mathrm{ZF})$, and minimum mean square error (MMSE). However, the measurements in Payami and Tufvesson (2013) were conducted in an outdoor environment. In this study, we focus on the performance of MF precoding for a massive MIMO channel. Based on the measurement data, we show that to which extent the RMS delay spread of the channel can be shortened when using an MF precoding scheme.

In the MF precoding scheme, the equivalent channel $H_{\mathrm{eq}}(f)$ which includes the combination of the precoder and the wireless channels in the frequency domain, was given by Payami and Tufvesson (2013) as

$$
H_{\mathrm{eq}}\left(f_{l}\right)=\sum_{i=1}^{N_{\mathrm{t}}} W_{i}\left(f_{l}\right) \cdot H_{i}\left(f_{l}\right),
$$

where $W_{i}$ is the precoding function of the $i$ th subchannel, $N_{\mathrm{t}}$ is the number of antennas at BS, and $f_{l}$ denotes the $l$ th frequency point. For simplicity, we analyze only the single user scenario; i.e., each $\mathrm{Rx}$ antenna is regarded as a single user. The precoding matrix $\boldsymbol{W}$ satisfies $\|\boldsymbol{W}\|=1$, which means

$$
\sqrt{\sum_{m=1}^{N_{\mathrm{t}}} \sum_{j=1}^{N_{\mathrm{u}}} \sum_{l=1}^{N_{\mathrm{f}}}\left|\boldsymbol{W}\left(m, j, f_{l}\right)\right|^{2}}=1,
$$

where $N_{\mathrm{u}}$ is the number of measured users. The precoding matrix can be calculated as (Payami and Tufvesson, 2013)

$$
\boldsymbol{W}\left(f_{l}\right)=b_{\mathrm{MF}}\left[\boldsymbol{H}\left(f_{l}\right)\right]^{\mathrm{H}},
$$

where $b_{\mathrm{MF}}$ is the normalization factor, $\boldsymbol{H}\left(f_{l}\right)$ is the $N_{\mathrm{u}} \times N_{\mathrm{t}}$ channel matrix at the frequency point $f_{l}$, and $[\cdot]^{\mathrm{H}}$ represents the Hermitian transposition. Using the equation above, MF precoding is performed at each frequency point $f_{l}\left(l=1,2, \cdots, N_{\mathrm{f}}\right)$ in the frequency domain. After that, the equivalent channel can be obtained and the CIR in the delay domain is found by using the inverse discrete Fourier transform. The RMS delay spread can be calculated as in Section 3.2. It should be stressed that our aim is to investigate the performance of the matched filter in the measured LOS/NLOS scenarios. Since the number of measured samples is limited, we randomly select $N_{\mathrm{t}}\left(N_{\mathrm{t}} \leq 64\right.$ or $\left.N_{\mathrm{t}} \leq 128\right)$ subsets from the total measurement data, corresponding to $N_{\mathrm{t}}$ measured subchannels. Then, we calculate the corresponding precoding matrix. These operations are repeated 400 times to obtain the RMS delay spreads of the equivalent channels for $M$ Tx antennas. Fig. 7 shows the performance of the matched filter at 2 and $22 \mathrm{GHz}$ in both LOS and NLOS scenarios. Space limitations prevent us from presenting similar results for the other frequencies we investigated. It is found that as the number of antennas at Tx increases, the RMS delay spread for the equivalent channel tends to be lower and less variable. The RMS delay spread corresponding to the probability $\mathrm{CDF}=0.5$ (computed by cumulative distribution function (CDF)) for the equivalent channel seems to converge to a value around $6.9 \mathrm{~ns}$ and $3.8 \mathrm{~ns}$ at 2 and $22 \mathrm{GHz}$, respectively.

Furthermore, it is noticed that the convergence rate becomes lower as the number of antennas at Tx increases, and having more than 32 antennas will not significantly contribute to channel shortening 

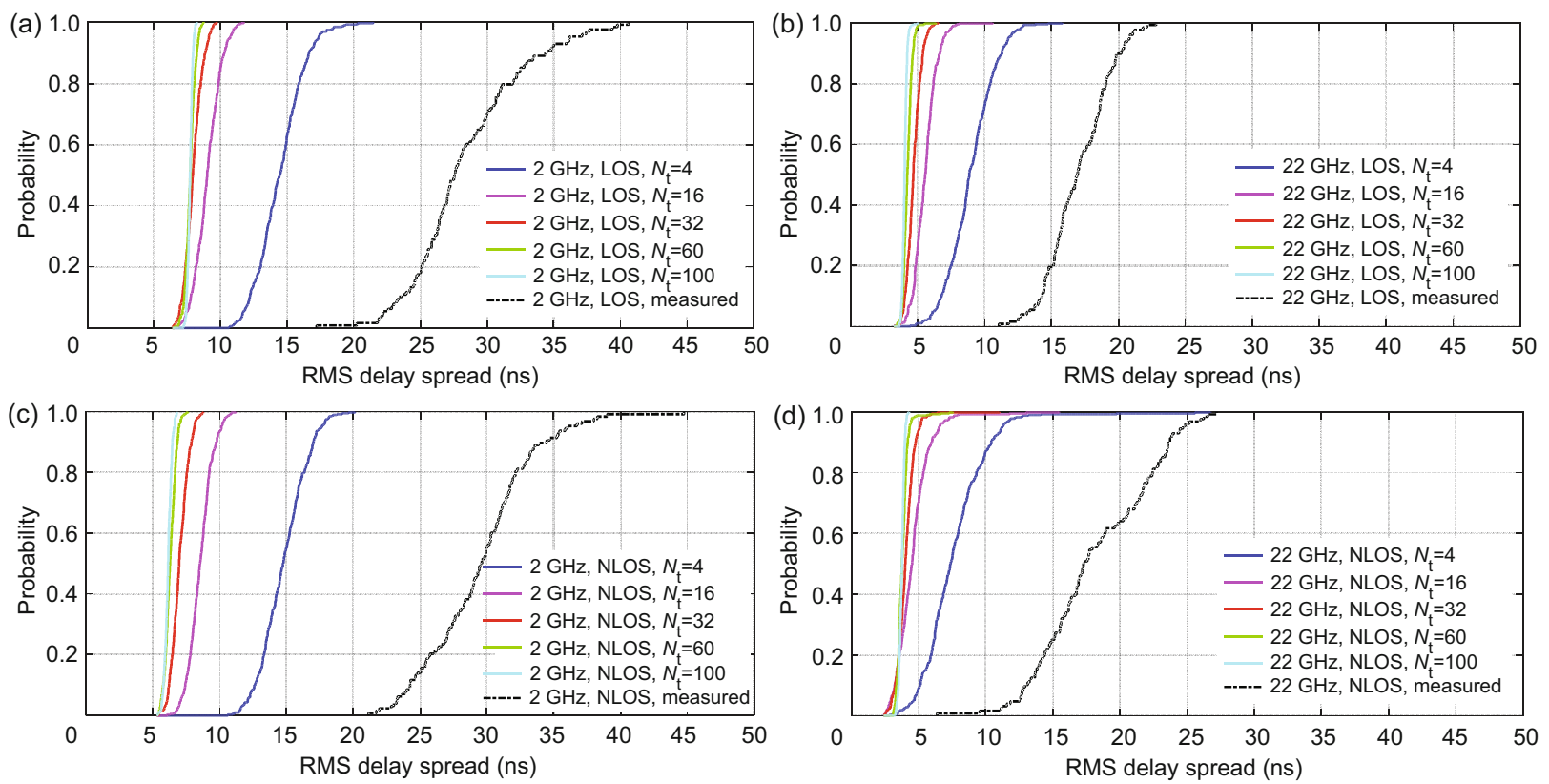

Fig. 7 Cumulative distribution functions of RMS delay spread for different numbers of Tx antennas with a 128-element planar array used at Tx: (a) at $2 \mathrm{GHz}$ in the LOS scenario; (b) at $22 \mathrm{GHz}$ in the LOS scenario; (c) at $2 \mathrm{GHz}$ in the NLOS scenario; (d) at $22 \mathrm{GHz}$ in the NLOS scenario (Tx: transmitter; RMS: root mean square; LOS: line-of-sight; NLOS: none-LOS)

effects. In addition, no frequency dependence is observed from the results. However, these results indicate that massive MIMO brings up new possibilities for performance improvements in wireless communication systems.

\subsection{Entropy capacity}

Entropy capacity $C$ can be derived from the channel transfer function $H(f)$ based on the measurement data. Assume that $\boldsymbol{H} \in \mathbb{C}^{N_{\mathrm{r}} \times N_{\mathrm{t}} \times N_{\mathrm{f}}}$ is the frequency domain massive MIMO channel matrix, where $N_{\mathrm{r}}$ is the number of $\mathrm{Rx}$ antennas. $\boldsymbol{H}_{l}$ is a matrix of $N_{\mathrm{r}}$ rows and $N_{\mathrm{t}}$ columns. It contains the $l$ th frequency sub-indexes for each measured subchannel. $H_{l}^{n m}$ denotes the element at the $n$th row and $m$ th column of $\boldsymbol{H}_{l}$. The average path gain $\eta$ of $\boldsymbol{H}$ can be calculated as (Jungnickel et al., 2009)

$$
\eta=\frac{1}{N_{\mathrm{t}} \cdot N_{\mathrm{r}} \cdot N_{\mathrm{f}}} \sum_{l=1}^{N_{\mathrm{f}}} \sum_{n=1}^{N_{\mathrm{r}}} \sum_{m=1}^{N_{\mathrm{t}}}\left|\boldsymbol{H}_{l}^{n m}\right|^{2} .
$$

We have $N_{\mathrm{r}}=4$ in the following evaluations. For each frequency index, a singular value decomposition of channel matrix $\boldsymbol{H}_{l}$ is performed:

$$
\boldsymbol{H}_{l}=\boldsymbol{U}_{l} \cdot \boldsymbol{D}_{l} \cdot \boldsymbol{V}_{l}^{\mathrm{H}},
$$

where $\boldsymbol{U}_{l}$ and $\boldsymbol{V}_{l}^{\mathrm{H}}$ are the unitary transform matrices. Diagonal matrix $\boldsymbol{D}_{l}$ contains $K=\min \left(N_{\mathrm{t}}, N_{\mathrm{r}}\right)$ nonzero singular values of $\sigma_{i, l}$ of $\boldsymbol{H}_{l}$, where $\sigma_{i}$ is the $i$ th singular value in matrix $\overline{\boldsymbol{H}}(f)$. The wideband entropy capacity is caculated as follows (Jungnickel et al., 2009):

$$
C=\frac{1}{N_{\mathrm{f}}} \sum_{l=1}^{N_{\mathrm{f}}} \sum_{i=1}^{K} \log _{2}\left(1+\frac{\rho}{N_{\mathrm{t}} \cdot \eta} \sigma_{i, l}^{2}\right),
$$

where $\rho$ denotes the signal-to-noise ratio (SNR). To investigate the impact of the measured channel on the capacity, $\rho$ is set to a constant value of $15 \mathrm{~dB}$ for all frequency bands, since it is the average SNR during the measurements. For simplicity, we assume that the channel is unknown to the transmitter, and that no water filling can be employed. We randomly select $N_{\mathrm{t}}\left(N_{\mathrm{t}} \leq 64\right)$ subsets from the total measurement data, corresponding to $N_{\mathrm{t}}$ measured subchannels. Then, we calculate the capacity of the selected MIMO channel and repeat this operation 400 times to obtain the entropy capacity for $N_{\mathrm{t}}$ Tx antennas.

Fig. 8 illustrates the example plots of the entropy capacity for a 64 -element planar Tx array at 2 and $22 \mathrm{GHz}$, respectively. It is hard to see any analogous frequency dependence of these results across the three types of array topologies. However, some 
(a)
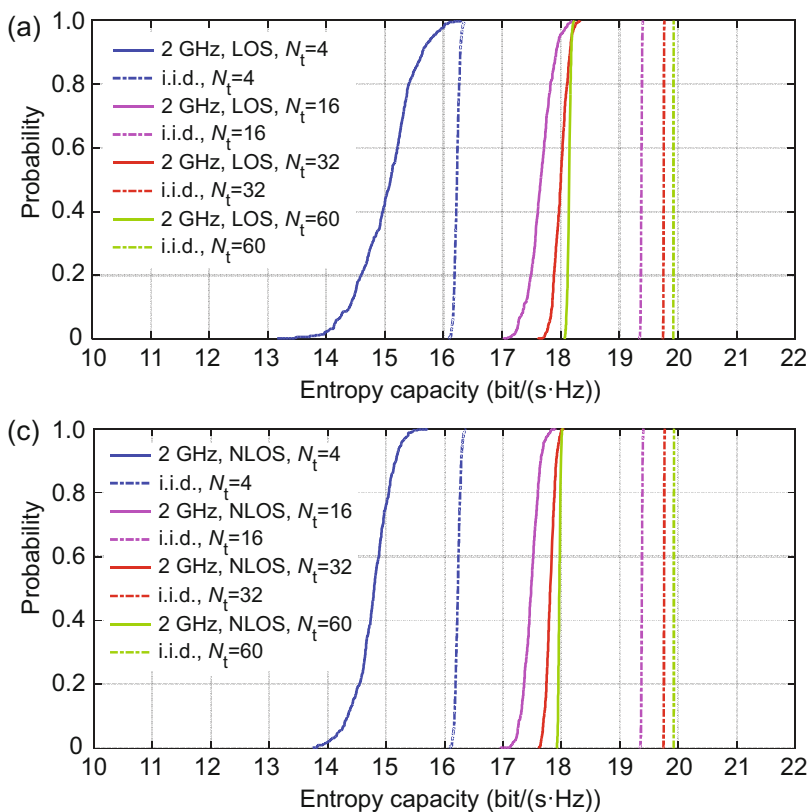
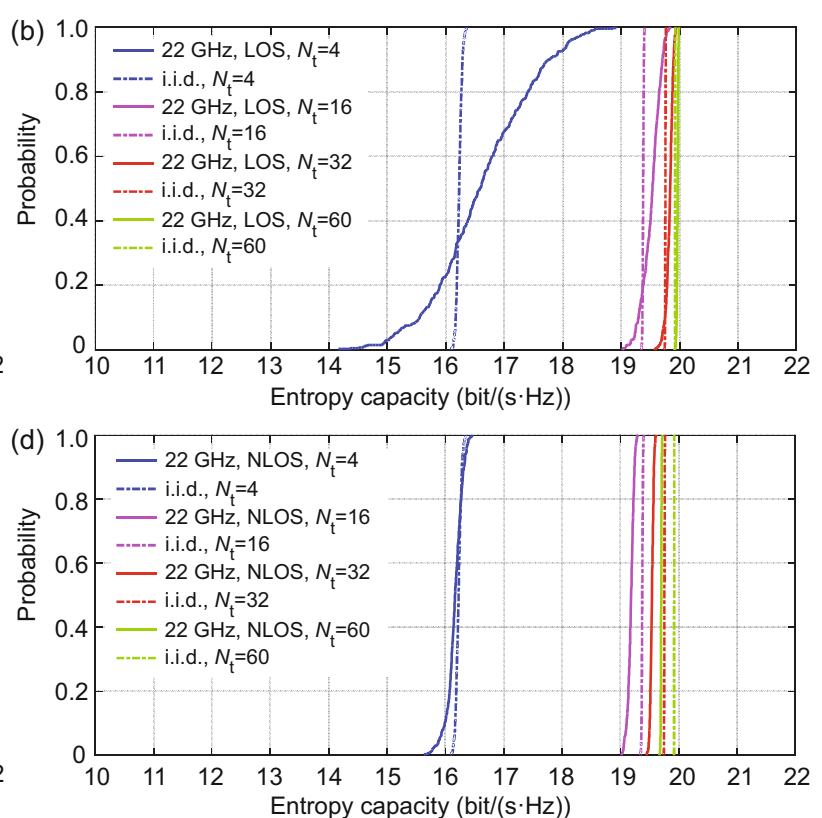

Fig. 8 Cumulative distribution functions of entropy capacity for different numbers of Tx antennas with a 64-element planar array used at Tx: (a) at $2 \mathrm{GHz}$ in the LOS scenario; (b) at $22 \mathrm{GHz}$ in the LOS scenario; (c) at $2 \mathrm{GHz}$ in the NLOS scenario; (d) at $22 \mathrm{GHz}$ in the NLOS scenario (Tx: transmitter; RMS: root mean square; LOS: line-of-sight; NLOS: none-LOS)

common characteristics can be found. As the number of antennas at Tx increases, the gap in capacity between the measured and i.i.d. channels becomes smaller. This phenomenon occurs more frequently at high frequencies and in NLOS scenarios. A propagation-based explanation for this phenomenon is that in such cases parallel information channels could arise more easily, and these parallel channels in space are mutually asymptotically orthogonal. This is beneficial in contributing a high channel capacity.

\subsection{Demmel condition number}

The Demmel condition number is a key indicator which measures the spatial multiplexing/ diversity performance of an MIMO channel. Heath and Paulraj (2005) set a threshold to compare it with the Demmel condition number, and to determine whether a spatial multiplexing scheme or diversity transmission scheme is employed. The Demmel condition number was defined in Demmel (1988) as

$$
\kappa_{\mathrm{D}}=\frac{\left\|\boldsymbol{H}_{l}\left(f_{l}\right)\right\|_{\mathrm{F}}}{\sigma_{\min }\left[\boldsymbol{H}_{l}\left(f_{l}\right)\right]},
$$

where $\sigma_{\min }\left[\boldsymbol{H}_{l}\left(f_{l}\right)\right]$ denotes the smallest singular value of matrix $\boldsymbol{H}_{l}\left(f_{l}\right)$. A large $\kappa_{\mathrm{D}}$ is suitable for diversity transmission, while a low $\kappa_{\mathrm{D}}$ is more suitable for spatial multiplexing, and $\kappa_{\mathrm{D}}=0$ corresponds to the ideal orthogonal channel case. For massive MIMO systems, spatial multiplexing is more concerned, since it substantially increases the throughput with the help of a large number of antennas at BS. Fig. 9 shows the CDFs of the Demmel condition number at 2 and $22 \mathrm{GHz}$ for 4, 16, 32, and $60 \mathrm{Tx}$ antennas. It can be seen that the Demmel condition number increases with the increase of the number of Tx antennas, indicating that the capability for spatial multiplexing of massive MIMO is stronger than that for traditional MIMO. Moreover, the capability for spatial multiplexing at $22 \mathrm{GHz}$ is slightly stronger than that at $2 \mathrm{GHz}$. These results are consistent with the estimated entropy capacity in Section 4.2.

\subsection{Ellipticity}

One of the basic ideas of MIMO/massive MIMO is that the anticipated throughput depends on the propagation environment providing parallel and independent channels to the terminals; thus, the multipath richness of the measured massive MIMO channel is a matter of concern in this study. Channel ellipticity as a measure of multipath richness was 

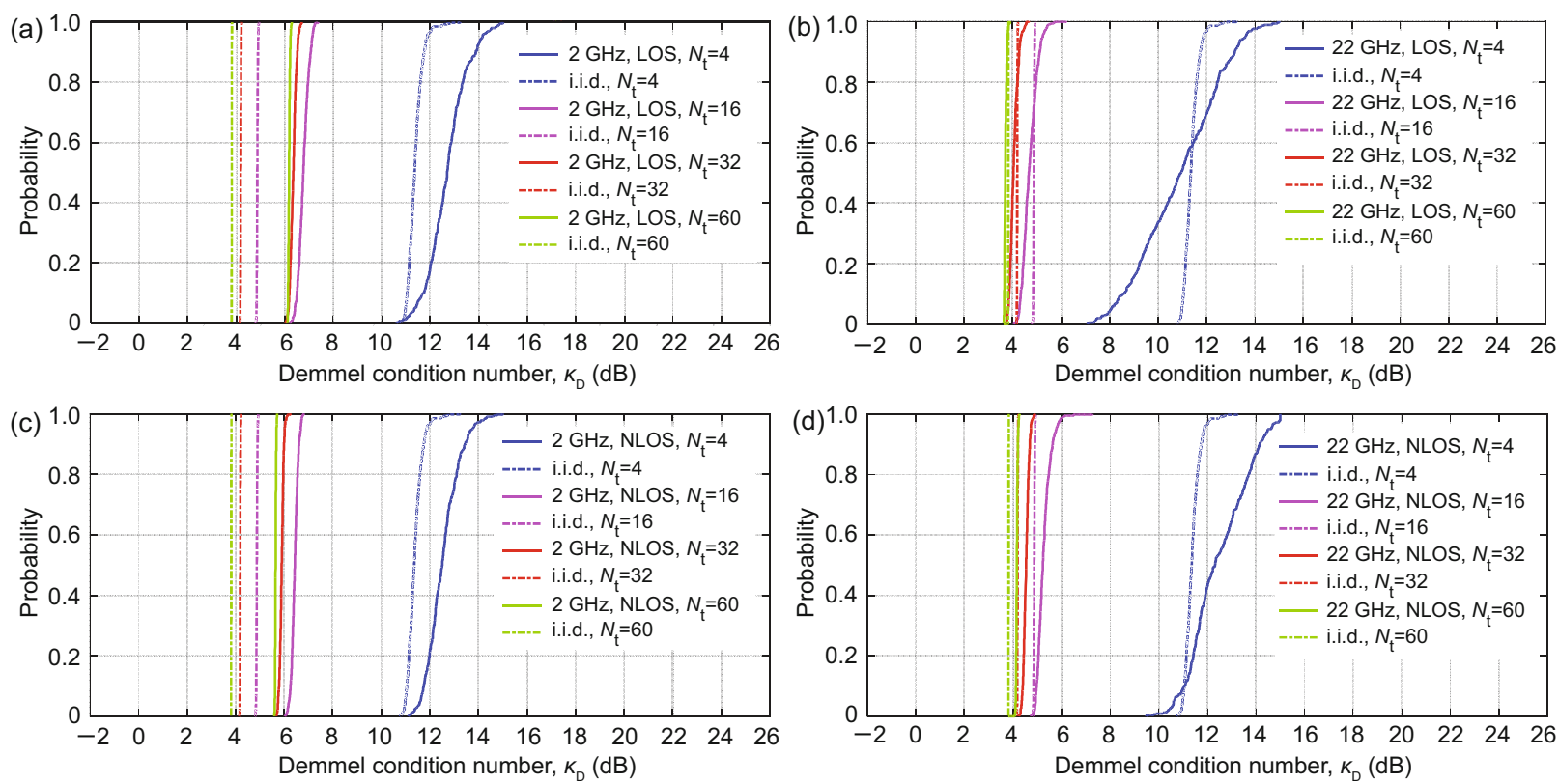

Fig. 9 Cumulative distribution functions of the Demmel condition number for different numbers of Tx antennas with a 64-element planar array used at Tx: (a) at 2 GHz in the LOS scenario; (b) at 22 GHz in the LOS scenario; (c) at $2 \mathrm{GHz}$ in the NLOS scenario; (d) at $22 \mathrm{GHz}$ in the NLOS scenario (Tx: transmitter; RMS: root mean square; LOS: line-of-sight; NLOS: none-LOS)

first introduced in Salo et al. (2006). The ellipticity of an MIMO channel indicates the information loss of the channel compared to a purely diagonal channel due to singular value dispersion (Demmel, 1988), defined as (Salo et al., 2006)

$$
\log _{2} \gamma=\log _{2}\left[\frac{\left(\prod_{i=1}^{K} \sigma_{i}\right)^{\frac{1}{K}}}{\frac{1}{K} \sum_{i=1}^{K} \sigma_{i}}\right],
$$

where $K=\min \left(N_{\mathrm{t}}, N_{\mathrm{r}}\right)$ equals 4 in our measurement since the $\mathrm{Rx}$ array is a 4-element array, and $\gamma$ is a function of all eigenvalues, more specifically, the ratio of arithmetic and geometric means of the channel eigenvalues, implying that this parameter is more suitable for measuring multipath richness compared with the Demmel condition number. From Eq. (17) we can see that channel ellipticity is always negative and gives the information loss in bits. The closer $\log _{2} \gamma$ is to 0 , the lower the capacity loss is, and $\log _{2} \gamma=0$ corresponds to the ideal orthogonal channel case.

Fig. 10 illustrates the CDFs of the channel ellipticity at 2 and $22 \mathrm{GHz}$ for $4,16,32$, and $60 \mathrm{Tx}$ antennas. It is observed that as the number of $\mathrm{Tx}$ antennas increases, the channel ellipticity increases, implying that the observed multipath richness can be improved by increasing the number of Tx antennas. In addition, channel ellipticity at $22 \mathrm{GHz}$ is observed to be larger than that at $2 \mathrm{GHz}$ for different numbers of antennas. Due to space limitations, we do not give all the other results for $4,6,11$, and $15 \mathrm{GHz}$ in this paper. However, it should be emphasized that these results are consistent with the estimated entropy capacity and the Demmel condition number.

\section{Conclusions}

In this paper, we presented an indoor measurement campaign for massive MIMO radio channels using a high-performance frequency domain channel sounder with different virtual large-scale arrays. The array types include a 64-element linear array, a 64-element planar array, and a 128-element planar array. The measurements were conducted at $2,4,6,11,15$, and $22 \mathrm{GHz}$, with a bandwidth of $200 \mathrm{MHz}$. Both LOS and NLOS propagation scenarios were considered. To generate different array topologies and guarantee the accuracy of the measurements, a high-precision 3D turntable was used. We also ensured that there were no moving people or other objects during the measurements. The highlights of our measurements are that pluralities of 

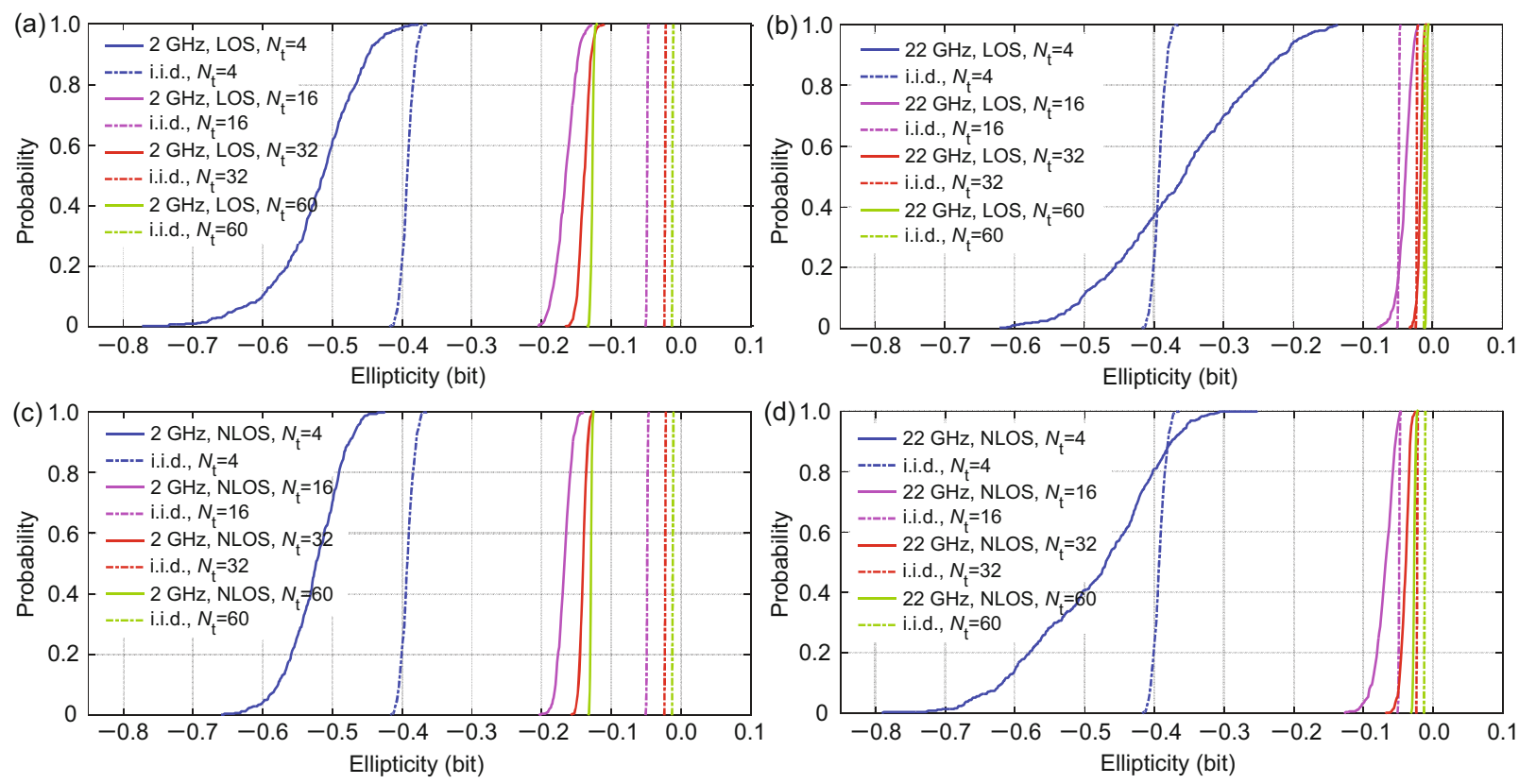

Fig. 10 Cumulative distribution functions of ellipticity for different numbers of Tx antennas with a 64-element planar array used at Tx: (a) at $2 \mathrm{GHz}$ in the LOS scenario; (b) at $22 \mathrm{GHz}$ in the LOS scenario; (c) at $2 \mathrm{GHz}$ in the NLOS scenario; (d) at $22 \mathrm{GHz}$ in the NLOS scenario (Tx: transmitter; RMS: root mean square; LOS: line-of-sight; NLOS: none-LOS)

frequency bands with a large bandwidth are measured, and different types of massive MIMO arrays are used. The basic channel parameters, including path loss, shadow fading, APDP, and RMS delay spread, were investigated based on the measurement data. We showed that the channel parameters vary significantly over the massive MIMO arrays, indicating that the channel characteristics of massive MIMO systems are non-stationary in both the spatial and delay domains. These characteristics should be taken into account when modeling massive MIMO channels. Moreover, the dependencies between these channel parameters and frequency were investigated. These results would be useful for the design of massive MIMO systems in indoor environments, and are expected to give a deeper insight into how massive MIMO performs in real propagation environments.

Furthermore, comprehensive performance evaluations based on realistic channels were carried out. The performance of the matched filter precoding scheme was investigated. Entropy capacity, Demmel condition number, and channel ellipticity were derived. It was shown that the performance of matched filter precoding can be improved by using more antennas in BS, and thus ISI can be minimized to the lowest level. On the other hand, as more antennas are used in BS, more parallel information channels exist in the environment, resulting in a high capacity. The subsequent analysis of the Demmel condition number and channel ellipticity further confirms this observation. The measured channels can achieve performance fairly close to that in i.i.d. Rayleigh channels even in an indoor LOS scenario.

\section{References}

Ai, B., Cheng, X., Kürner, T., et al., 2014. Challenges toward wireless communications for high-speed railway. IEEE Trans. Intell. Transp. Syst., 15(5):2143-2158. http://dx.doi.org/10.1109/TITS.2014.2310771

Ai, B., Guan, K., Rupp, M., et al., 2015. Future railway services-oriented mobile communications network. IEEE Commun. Mag., 53(10):78-85. http://dx.doi.org/10.1109/MCOM.2015.7295467

Ai, B., Guan, K., He, R.S., et al., 2017. On indoor millimeter wave massive MIMO channels: measurement and simulation. IEEE J. Sel. Areas Commun., 99:1-17. http://dx.doi.org/10.1109/JSAC.2017.2698780

Andrews, J.G., Buzzi, S., Choi, W., et al., 2014. What will 5G be? IEEE J. Sel. Areas Commun., 32(6):1065-1082. http://dx.doi.org/10.1109/JSAC.2014.2328098

Astely, D., Dahlman, E., Furuskär, A., et al., 2009. LTE: the evolution of mobile broadband. IEEE Commun. Mag., 47(4):44-51.

http://dx.doi.org/10.1109/MCOM.2009.4907406

Boccardi, F., Heath, R.W., Lozano, A., et al., 2014. Five disruptive technology directions for $5 \mathrm{G}$. IEEE Commun. Mag., 52(2):74-80.

http://dx.doi.org/10.1109/MCOM.2014.6736746 
Cai, Y., de Lamare, R.C., Champagne, B., et al., 2015. Adaptive reduced-rank receive processing based on minimum symbol-error-rate criterion for large-scale multiple-antenna systems. IEEE Trans. Commun., 63(11):4185-4201.

http://dx.doi.org/10.1109/TCOMM.2015.2475260

Demmel, J.W., 1988. The probability that a numerical analysis problem is difficult. Math. Comput., 50(182):449480.

http://dx.doi.org/10.1090/S0025-5718-1988-0929546-7

Feuerstein, M.J., Blackard, K.L., Rappaport, T.S., et al., 1994. Path loss, delay spread, and outage models as functions of antenna height for microcellular system design. IEEE Trans. Veh. Technol., 43(3):487-498. http://dx.doi.org/10.1109/25.312809

Flordelis, J., Gao, X., Dahman, G., et al., 2015. Spatial separation of closely-spaced users in measured massive multi-user MIMO channels. IEEE Int. Conf. on Communications, p.1441-1446.

http://dx.doi.org/10.1109/ICC.2015.7248526

Gao, L., Zhong, Z., Ai, B., et al., 2010. Estimation of the Ricean $K$ factor in the high speed railway scenarios. 5th Int. Conf. on Communications and Networking in China, p.1-5.

Gao, X., Tufvesson, F., Edfors, O., et al., 2012. Measured propagation characteristics for very-large MIMO at 2.6 GHz. 46th Asilomar Conf. on Signals, Systems and Computers, p.295-299. http://dx.doi.org/10.1109/ACSSC.2012.6489010

Gao, X., Edfors, O., Rusek, F., et al., 2015. Massive MIMO performance evaluation based on measured propagation data. IEEE Trans. Wirel. Commun., 14(7):3899-3911. http://dx.doi.org/10.1109/TWC.2015.2414413

Greenstein, L.J., Erceg, V., Yeh, Y.S., et al., 1997. A new path-gain/delay-spread propagation model for digital cellular channels. IEEE Trans. Veh. Technol., 46(2):477-485. http://dx.doi.org/10.1109/25.580786

Guan, K., Zhong, Z., Ai, B., et al., 2013a. Deterministic propagation modeling for the realistic high-speed railway environment. IEEE 77th Vehicular Technology Conf., p.1-5.

http://dx.doi.org/10.1109/VTCSpring.2013.6692506

Guan, K., Zhong, Z., Ai, B., et al., 2013b. Modeling of the division point of different propagation mechanisms in the near-region within arched tunnels. Wirel. Pers. Commun., 68(3):489-505.

http://dx.doi.org/10.1007/s11277-011-0464-7

Guan, K., Zhong, Z., Ai, B., et al., 2014a. Propagation measurements and analysis for train stations of highspeed railway at $930 \mathrm{MHz}$. IEEE Trans. Veh. Technol., 63(8):3499-3516. http://dx.doi.org/10.1109/TVT.2014.2307917

Guan, K., Zhong, Z., Ai, B., et al., 2014b. Propagation measurements and modeling of crossing bridges on highspeed railway at $930 \mathrm{MHz}$. IEEE Trans. Veh. Technol., 63(2):502-517. http://dx.doi.org/10.1109/TVT.2013.2275912

Guan, K., Ai, B., Nicolás, M.L., et al., 2016. On the influence of scattering from traffic signs in vehicle-to- $x$ communications. IEEE Trans. Veh. Technol., 65(8):5835-5849. http://dx.doi.org/10.1109/TVT.2015.2476335
He, R., Zhong, Z., Ai, B., et al., 2011. An empirical path loss model and fading analysis for high-speed railway viaduct scenarios. IEEE Antennas Wirel. Propag. Lett., 10:808-812. http://dx.doi.org/10.1109/LAWP.2011.2164389

He, R., Zhong, Z., Ai, B., et al., 2012a. Analysis of the relation between Fresnel zone and path loss exponent based on two-ray model. IEEE Antennas Wirel. Propag. Lett., 11:208-211. http://dx.doi.org/10.1109/LAWP.2012.2187270

He, R., Zhong, Z., Ai, B., et al., 2012b. Measurements and analysis of short-term fading behavior for highspeed rail viaduct scenario. IEEE Int. Conf. on Communications, p.4563-4567. http://dx.doi.org/10.1109/ICC.2012.6363678

He, R., Zhong, Z., Ai, B., et al., 2013. Measurements and analysis of propagation channels in high-speed railway viaducts. IEEE Trans. Wirel. Commun., 12(2):794805. http://dx.doi.org/10.1109/TWC.2012.120412.120268

He, R., Molisch, A.F., Tufvesson, F., et al., 2014. Vehicle-tovehicle propagation models with large vehicle obstructions. IEEE Trans. Intell. Transp. Syst., 15(5):22372248. http://dx.doi.org/10.1109/TITS.2014.2311514

He, R., Renaudin, O., Kolmonen, V.M., et al., 2015a. Characterization of quasi-stationarity regions for vehicle-tovehicle radio channels. IEEE Trans. Antennas Propag., 63(5):2237-2251. http://dx.doi.org/10.1109/TAP.2015.2402291

He, R., Zhong, Z., Ai, B., et al., 2015b. Shadow fading correlation in high-speed railway environments. IEEE Trans. Veh. Technol., 64(7):2762-2772. http://dx.doi.org/10.1109/TVT.2014.2351579

He, R., Ai, B., Wang, G., et al., 2016a. High-speed railway communications: from GSM-R to LTE-R. IEEE Veh. Technol. Mag., 11(3):49-58. http://dx.doi.org/10.1109/MVT.2016.2564446

He, R., Chen, W., Ai, B., et al., 2016b. On the clustering of radio channel impulse responses using sparsity-based methods. IEEE Trans. Antennas Propag., 64(6):24652474. http://dx.doi.org/10.1109/TAP.2016.2546953

Heath, R.W., Paulraj, A.J., 2005. Switching between diversity and multiplexing in MIMO systems. IEEE Trans. Commun., 53(6):962-968. http://dx.doi.org/10.1109/TCOMM.2005.849774

Hoydis, J., Hoek, C., Wild, T., et al., 2012. Channel measurements for large antenna arrays. Int. Symp. on Wireless Communication Systems, p.811-815. http://dx.doi.org/10.1109/ISWCS.2012.6328480

Janssen, G.J.M., Stigter, P.A., Prasad, R., 1996. Wideband indoor channel measurements and BER analysis of frequency selective multipath channels at $2.4,4.75$, and 11.5 GHz. IEEE Trans. Commun., 44(10):1272-1288. http://dx.doi.org/10.1109/26.539768

Jungnickel, V., Jaeckel, S., Thiele, L., et al., 2009. Capacity measurements in a cooperative MIMO network. IEEE Trans. Veh. Technol., 58(5):2392-2405. http://dx.doi.org/10.1109/TVT.2008.2010260

Larsson, E.G., Edfors, O., Tufvesson, F., et al., 2014. Massive MIMO for next generation wireless systems. IEEE Commun. Mag., 52(2):186-195. http://dx.doi.org/10.1109/MCOM.2014.6736761 
Li, J., Ai, B., He, R., et al., 2016. Measurement-based characterizations of indoor massive MIMO channels at $2 \mathrm{GHz}, 4 \mathrm{GHz}$, and $6 \mathrm{GHz}$ frequency bands. IEEE 83rd Vehicular Technology Conf., p.1-5. http://dx.doi.org/10.1109/VTCSpring.2016.7504341

Liu, L., Li, Y., Zhang, J., 2014. DoA estimation and achievable rate analysis for 3D millimeter wave massive MIMO systems. IEEE 15th Int. Workshop on Signal Processing Advances in Wireless Communications, p.610. http://dx.doi.org/10.1109/SPAWC.2014.6941306

Molisch, A.F., 2011. Wireless Communications. Wiley-IEEE Press, Hoboken, USA.

Molisch, A.F., Steinbauer, M., 1999. Condensed parameters for characterizing wideband mobile radio channels. Int. J. Wirel. Inform. Netw., 6(3):133-154. http://dx.doi.org/10.1023/A:1018895720076

Ng, B.L., Kim, Y., Lee, J., et al., 2012. Fulfilling the promise of massive MIMO with 2D active antenna array. IEEE Globecom Workshops, p.691-696. http://dx.doi.org/10.1109/GLOCOMW.2012.6477658

Ngo, H.Q., Larsson, E.G., Marzetta, T.L., 2013. Energy and spectral efficiency of very large multiuser MIMO systems. IEEE Trans. Commun., 61(4):1436-1449. http://dx.doi.org/10.1109/TCOMM.2013.020413.110848

Payami, S., Tufvesson, F., 2012. Channel measurements and analysis for very large array systems at $2.6 \mathrm{GHz}$. 6th European Conf. on Antennas and Propagation, p.433437. http://dx.doi.org/10.1109/EuCAP.2012.6206345

Payami, S., Tufvesson, F., 2013. Delay spread properties in a measured massive MIMO system at $2.6 \mathrm{GHz}$. IEEE 24th Annual Int. Symp. on Personal, Indoor, and Mobile Radio Communications, p.53-57. http://dx.doi.org/10.1109/PIMRC.2013.6666103
Poon, A.S.Y., Ho, M., 2003. Indoor multiple-antenna channel characterization from 2 to $8 \mathrm{GHz}$. IEEE Int. Conf. on Communications, p.3519-3523. http://dx.doi.org/10.1109/ICC.2003.1204108

Rusek, F., Persson, D., Lau, B.K., et al., 2013. Scaling up MIMO: opportunities and challenges with very large arrays. IEEE Signal Process. Mag., 30(1):40-60. http://dx.doi.org/10.1109/MSP.2011.2178495

Salo, J., Suvikunnas, P., El-Sallabi, H.M., et al., 2006. Ellipticity statistic as measure of MIMO multipath richness. Electron. Lett., 42(3):160-162. http://dx.doi.org/10.1049/el:20063847

Salous, S., Gokalp, H., 2007. Medium- and large-scale characterization of UMTS-allocated frequency division duplex channels. IEEE Trans. Veh. Technol., 56(5):28312843. http://dx.doi.org/10.1109/TVT.2007.900495

Wang, C.X., Haider, F., Gao, X., et al., 2014. Cellular architecture and key technologies for $5 \mathrm{G}$ wireless communication networks. IEEE Commun. Mag., 52(2):122-130. http://dx.doi.org/10.1109/MCOM.2014.6736752

Wei, H., Zhong, Z., Xiong, L., et al., 2011. Study on the shadow fading characteristic in viaduct scenario of the high-speed railway. 6th Int. Conf. on Communications and Networking in China, p.1216-1220.

http://dx.doi.org/10.1109/ChinaCom.2011.6158343

Wu, S., Wang, C.X., Haas, H., et al., 2015. A non-stationary wideband channel model for massive MIMO communication systems. IEEE Trans. Wirel. Commun., 14(3):1434-1446.

http://dx.doi.org/10.1109/TWC.2014.2366153 